\title{
Carriage of Staphylococcus Aureus in Psoriasis
}

\author{
W. C. NOBLE,* M.SG., PH.D. ; J. A. SAVIN, $\dagger$ M.A., M.B., M.R.C.P., D.I.H.
}

Brit. med. F., 1968, 1, 417-419

Bacteriological studies have suggested that psoriatic plaques are often colonized by Staphylococcus aureus without clinical evidence of infection (Cooke and Buck, 1963 ; Selwyn, 1965 ; Selwyn and Chalmers, 1965). The recent report of an outbreak of staphylococcal wound infection traced to an anaesthetist with psoriasis supports this and emphasizes the importance of unrecognized carriage of pathogens (Payne, 1967). Though patients with psoriasis may themselves be unusually resistant to skin sepsis (Ingram, 1964), it has been suggested that for the safety of others they should not be admitted to general wards until they have been bacteriologically screened (Lancet, 1965). As psoriatics may account for one admission in 50 this is of practical importance (Ingram, 1964).

In this study we tried to find out whether psoriatic plaques were selectively colonized with Staph. aureus by comparing their flora with that of adjacent normal skin.

\section{Methods}

The best way of sampling the skin flora quantitatively is to use a brush rotating in a cup of broth pressed against the skin (Pillsbury and Nichols, 1946). In psoriasis, however, the abnormal horny layer is easily detached as scales, and would probably not respond to scrubbing in the same way as normal skin. The quantity of scale removed would depend, among other things, on how recently the plaque had been cleaned. For this reason, and because the brush method is rather cumbersome in practice, it was not used in the present study.

The use of contact plates for sampling the skin surface is very convenient and its reproducibility has been validated (Ulrich, 1965). We used the lids of 2-in. (5-cm.) Petri dishes filled so that the meniscus stood above the edge of the plastic. The medium used was Oxoid blood agar base containing $5 \%$ horse serum ; all plates were incubated at $37^{\circ} \mathrm{C}$. aerobically, as the main interest lay in the isolation of Staph. aureus. Two plates were used for each sample, the agar being pressed firmly against the skin surface for three seconds to ensure contact. In patients with psoriasis one plate was pressed on the plaque and the other on adjacent normal skin. Where possible, plaques about $1 \frac{1}{2}$ in. $(3.8 \mathrm{~cm}$.) in diameter were chosen for sampling, flexural areas being avoided. In addition, we tried to assess the validity of the method by studying differences between pairs of samples taken from normal skin of patients with diseases other than psoriasis or eczema and of clinically normal controls from among laboratory staff ; in each pair sampled the first sample was compared with the second. This method does not, of course, give information on the "clump size" but records the number of colony-forming units on the skin surface.

\section{Results}

Validation of Method.-The total counts of bacteria on each pair of plates from normal skin agreed well whether the arithmetic or logarithmic values were considered (see Table), but the distribution conformed more closely to the logarithmic

- St. John's Hospital for Diseases of the Skin, London W.C.2.

t St. John's Hospital for Diseases of the Skin, London W.C.2, and Department of Dermatology, St. George's Hospital, London S.W.1. count. Two adjacent areas of skin might be expected to support a similar population unless one is a special growth site or lesion. In this case the correlation line between the two samples of each pair would run equidistant from the axis. Fig. 1 shows the theoretical and actual correlation lines and the $95 \%$ confidence limits for the normal samples. The pairs of samples from normal skin correspond very closely to the theoretical expectation.

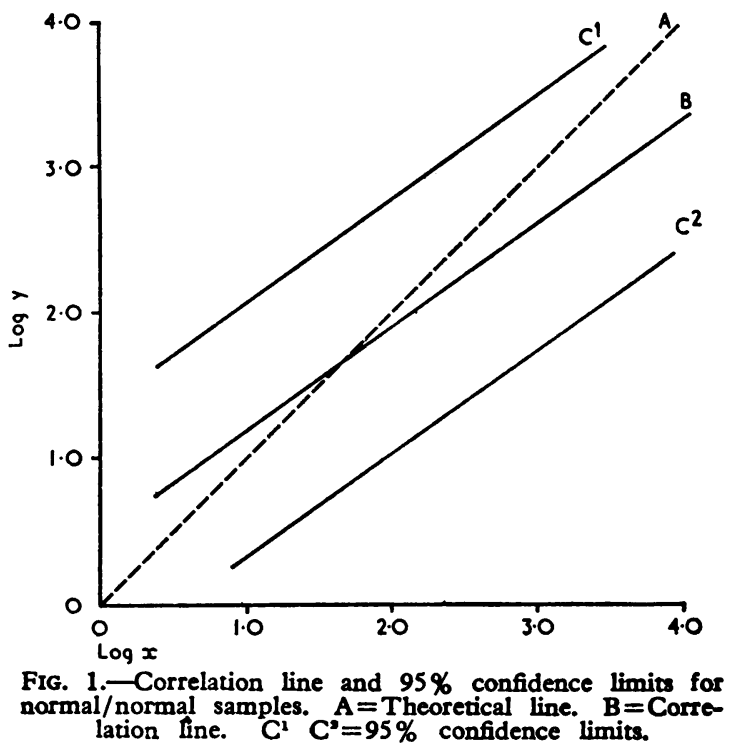

\section{Comparison of Plaques with Normal Skin}

A total of 111 pairs of samples were taken from 34 patients with psoriasis, of whom 16 were inpatients. In view of the results obtained with normal skin, a logarithmic distribution was used for analysis of the sample pairs. Nine of the 34 patients yielded Staph. aureus from one or more samples; seven of the nine were inpatients. There was a good correlation between the pairs of samples, even when the arithmetic values for only those samples yielding Staph. aureus were considered (see Table). The mean arithmetic counts of Staph. aureus were 30 colonies on the plaque samples and 10 on the normal skin, but these means were not significantly different $(t=1.97,10 \%>P>5 \%, n=27)$.

Statistical Values for Correlation Coefficionts

\begin{tabular}{|c|c|c|c|c|c|c|c|}
\hline \multirow{2}{*}{ Analysis } & \multirow{2}{*}{ Values } & \multirow{2}{*}{$\begin{array}{l}\text { Cor- } \\
\text { relation } \\
\text { Coeff- } \\
\text { cient }\end{array}$} & \multirow{2}{*}{$\begin{array}{l}\text { Total } \\
\text { Pairs }\end{array}$} & \multicolumn{2}{|c|}{ Mean } & \multirow{2}{*}{ S.D. } & \multirow{2}{*}{$\begin{array}{c}\text { S.D. as } \\
\% \text { of } \\
\text { Mean } \\
\text { Count }\end{array}$} \\
\hline & & & & 1 & 2 & & \\
\hline \multirow{3}{*}{$\begin{array}{l}\text { Normal } v . \\
\text { normal } \\
\text { skin } \\
\text { Normal } v . \\
\text { psoriatic } \\
\text { plaques }\end{array}$} & \multirow{3}{*}{$\begin{array}{l}\text { Arithmetic } \\
\text { Logarith- } \\
\text { mic } \\
\text { Logarith- } \\
\text { mic }\end{array}$} & $0.95^{*}$ & 65 & 180 & 171 & 122 & 68 \\
\hline & & 0.72 & 65 & 1.74 & $1 \cdot 72$ & 0.4 & 23 \\
\hline & & $0.79 *$ & 111 & 1.67 & 1.62 & 0.6 & 37 \\
\hline \multirow{2}{*}{$\begin{array}{l}\text { Biased } \\
\text { sample of } \\
\text { Staph. } \\
\text { curreus } \\
\text { only }\end{array}$} & $\begin{array}{l}\text { Logarith- } \\
\text { mic }\end{array}$ & $0.71 *$ & 27 & 0.57 & 0.68 & 0.59 & 95 \\
\hline & Arithmetic & $0.54 t$ & 27 & $10 \cdot 0$ & 30.5 & $44 \cdot 5$ & 217 \\
\hline
\end{tabular}

- Significant below $0.1 \%$ level. + Significant between $1 \%$ and $0.1 \%$ levels. 
In view of the small numbers of staphylococci isolated in the study, statistical analyses were also carried out on the total skin flora. Again, there was a statistically significant correlation between pairs (see Table).

There were no significant differences between the correlation lines for normal/psoriatic and normal/normal samples $(t=0.58$, P>10\%).

The logarithmic and arithmetic distributions differed markedly for the total skin flora. With the logarithmic distribution the mean counts on the plaque and normal skin were almost identical (1.67 and 1.62 respectively). With the arithmetic distribution, however, the mean plaque count (322) was greater than the mean normal count (222). From the data it is clear that this was the result of a few very high counts on the plaque, the majority conforming closely to the "expected." This is also true of the counts for Staph. aureus. The differences in the arithmetic means are in this case due to a few high counts from two individuals only.

Ratio Between Samples.-Another way of analysing these results is to consider the ratio of the count between normal skin and the plaque. In this series the count was greater on the normal skin 57 times, greater on the plaque 49 times, and equal 5 times in the 111 samples.

There was no significant difference between the normal skin of psoriatics and that of non-psoriatics $(t=1.2, P>10 \%, n=$ 239) ; the data from normal persons were therefore pooled with

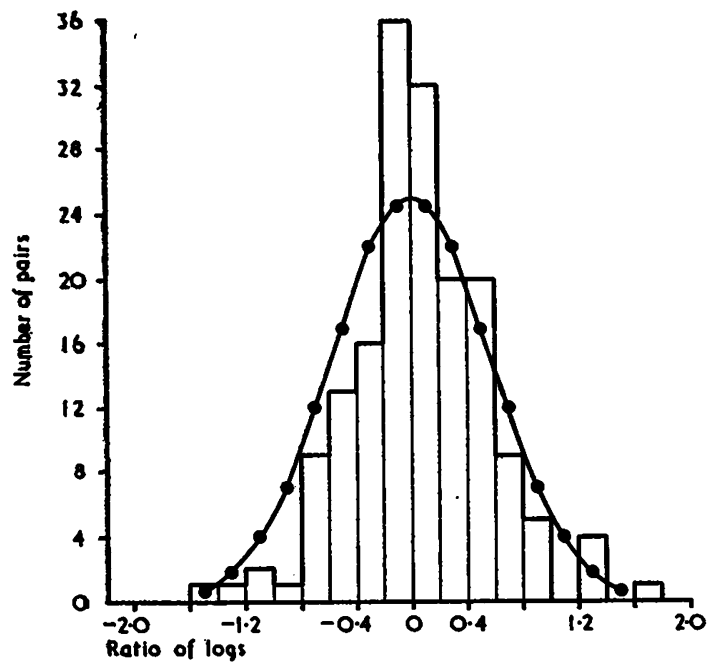

Fro. 2.-Distribution histogram of $\log$ ratios and normal distribution curve with same mean and standard

those of the psoriatics. The distribution of the logarithms of the ratios was obtained, the normal skin or first sample being used as the index in each case. Fig. 2 shows the distribution of these logarithm ratios and the normal distribution curve with the same mean and standard deviation. It is clear from this that the distribution of ratios corresponds closely to the normal distribution, and the occasional excessively high or low ratios encountered between normal skin and plaques were part of the normal range of results.

\section{Discussion}

Our findings were unexpected, as the idea that psoriatic plaques may be heavily colonized by staphylococci had seemed a reasonable one. A plaque, with its obvious disturbance of normal skin structure, might well have lacked some of the factors which normally help to rid the skin of bacteria. Psoriatics may be very prolific dispersers of particles bearing staphylococci (Thomas and Griffiths, 1961 ; Selwyn and Chalmers, 1965 ; Payne, 1967). The rapid epidermal turnover time may cause increased shedding of small particles of skin as well as the rather large particles usually associated with psoriasis (Rothberg, Crounse, and Lee, 1961).

Only 9 of our 34 patients yielded Staph. asceus; seven of the nine were inpatients. The difference between the recovery of Staph. aureus from 7 of the 16 inpatients but from only 2 of the 18 outpatients is significant between the $2 \%$ and $1 \%$ levels $\left(x^{2}=5.8 \%\right)$. As the flora of the plaques did not differ significantly from that of the adjacent normal skin, the carriage of Staph. aureus may merely reflect the environment in which the patient is sampled rather than a characteristic of the disease. The opportunities for acquiring staphylococci are much greater in a hospital ward than outside it.

It may be significant that although the anaesthetist described by Payne (1967) had had psoriasis for eight years, it was only after he had been in hospital for treatment that he was found to have become a disperser of staphylococci.

In this study 7 (44\%) of the 16 inpatients yielded Staph. aureus from plaques or adjacent normal skin. Selwyn (1965) recorded that $35 \%$ of 114 patients with psoriasis became colonized in the wards, though he does not state whether skin other than lesions was involved. In the course of other investigations in dermatological wards (Noble, 1966) $28 \%$ of 36 patients with psoriasis yielded Staph. aureus from uninvolved areas of the chest and groin. Outpatients with psoriasis seldom yield Staph. aureus (Selwyn, 1965 ; Noble, unpublished observations). Though it is clear that a patient with an infected psoriatic lesion may prove a serious hazard in a surgical ward, this seems likely to be a reflection of the hospital environment rather than a characteristic of the disease.

\section{Summary}

A study of the aerobic bacterial flora of psoriatic plaques and adjacent normal skin, using contact plates, showed no significant difference in the carriage of either total flora or of Staphylococcus aureus. Occasional very high or very low counts appeared to be part of the normal distribution. More inpatients than outpatients carried Staph. aureus; this seems to be a reflection of the hospital environment.

We are indebted to the consultant staft of St. John's Hospital and St. George's Hospital for permission to examine patients under their care.

\section{REFERENCES}

Cooke, E. Mary, and Buck, H. W. (1963). Brit. 7. Deren., 75, 21. Ingram, J. T. (1964). Lancet, 1, 121.

Lancet, 1965, 2, 483 .

Noble, W. C. (1966). 7. clin. Path., 19, 570.

Payne, R. W. (1967). Brit. med. F., 4, 17.

Pillsbury, D. M., and Nichols, A. C. (1946). F. invess, Dorm., 7, 365.

Rothberg, S., Crounse, R. G., and Lee, J. L. (1961). Ibid., 37, 497.

Selwyn, S. (1965). F. Hyg. (Lond.), 63, 59.

and Chalmers, D. (1965), Brit. 7. Derm., 77, 349.

Thomas, C. G. A., and Griffiths, P. D. (1961). Guy's Hosp. Rep., 110, 76.

Ulrich, J. A. (1965). In Skin Bacteria and their Role in Infection, edited by H. I. Maibach and G. Hildick-Smith, p. 219. New York. 